\title{
Edge-Preserving Decompositions for Multi-Scale with Adaptive TV
}

\author{
Cui Zhiqiang ${ }^{1}$ and Su Zhixun ${ }^{2}$ \\ ${ }^{1)}$ School of Mathematical Sciences, Dalian University of Technology, Dalian, Liaoning, China (czq_maths@ @ail.dlut.edu.cn) \\ ${ }^{2)}$ School of Mathematical Sciences, Dalian University of Technology, Dalian, Liaoning, China (zxsu@dlut.edu.cn)
}

Abstract-Digital image processing has received widespread attention in recent years, multi-scale image decomposition has been extensively studied. Many recent computational photography techniques decompose an image into a smooth base layer, and a residual detail layer. In many applications, it is important to control the spatial scale of the extracted details.

Aiming at this problem, we have extensively studied the weighted least squares framework firstly, through the image priors total variation to preserve the edge; Second, the paper develop an adaptive weight function that is able to describe some structures of an image. This adaptive weight function together with TV regularizer can able preserve large scale structures and remove tiny details. Finally, the proposed method is solved by alternating iterative minimization, which has fast convergence peroperty.

Experiments show that our method can keep the image edges and avoid visual artifacts in image processing. Moreover, our method has obvious advantages in operation time. It is efficient algorithm to extracted details at the arbitrary multi-scale.

Keywords —Edge-Preserving, WLS, adaptive TV, ADM, Multi-Scale Decomposition

\section{基于局部加权 TV 的保边界平滑及多尺度分解}

\author{
崔志强 ${ }^{1}$ 苏志勋 $^{2}$ \\ 1) 大连理工大学数学科学学院计算几何与图形图像实验室, 大连, 辽宁, 中国 \\ 2) 大连理工大学数学科学学院计算数学所, 大连, 辽宁, 中国
}

\begin{abstract}
摘 要 近年来, 数字图像处理受到越来越多的关注。作为图像处理中的基础部分, 图像多尺度分解问题一直是国际上的研究热 点。在图像分解方法中, 最常见的是将图像平滑得到基础层, 然后将原图像与基础层相减得到细节层。对许多应用来说, 图像分解最 重要的是通过空间尺度来提取出所需尺度的信息。

本文针对传统的保边界平滑算法, 如双边滤波方法(Bilateral Filter;BLF)、加权最小二乘方法(Weighted Least Squares;WLS)提出基 于图像的全变差约束(Total Variation;TV)来保持图像边界; 其次, 本文使用了一种区域化的梯度先验函数来保持结构信息并去掉细节信 息。最后本文使用交替方向迭代算法(Alternating Direction Method;ADM)使得局部加权的 TV 方法(adaptive TV)可解并实现其快速算法。 实验表明, 基于图像的局部加权的 TV 方法(adaptive TV)可以更好的保持边界, 减少边界处的光晕现象; 在运算时间上也有明显优 势; 对图像的任意尺度的细节都能很好地提取, 是一种有效的算法。
\end{abstract}

关键词＼cjkstart保边界平滑，加权最小二乘，局部加权 TV，交替迭代，多尺度分解

\section{1. 引言}

图像的多尺度分解问题近年来得到越来越多的关注, 成为了数字图像处理中的热点问题。图像的多尺度分解一 般是将图像平滑得到基础层, 然后将原图像与基础层相减

国家自然科学基金项目支持（资助号：61173103）
得到细节层。这种分解为 HDR 色调映射问题(HDR tone mapping)[1], 闪光图像融合问题 (flash/no-flash image fusion)[2], 图像编辑(image editing)[3], 或者其他类似问题 提供基础。

传统上, 对于图像的多尺度分解使用拉普拉斯金字塔 方法(the Laplacian pyramid) ${ }^{[4]}$ 。然而, 由于该方法使用线性 
滤波器, 会在图像边界处产生严重的光晕现象。为了减少 光晕现象, 人们开始使用非线性的滤波器。双边滤波器(BLF) 成为处理图像的保边界平滑中最流行的方法, 它对降低噪 声和小细节提取有很好的表现, 但是缺乏对任意尺度细节 提取的能力。为了适合任意尺度细节的提取, Farbman ${ }^{[5]}$ 等人提出了加权最小二乘方法(WLS), 他们使用一种特殊 的各项异性约束来对原图像进行平滑。为了更好的刻画图 像的梯度分布, 本文使用图像的全变差约束(TV, 即使用各 向异性 $\mathrm{L}^{1}$ 范数) 以及一种区域化的原图像梯度先验函数。 本文的组织如下：第一节问题的介绍。第二节问题的背景 知识和相关工作, 重点研究双边滤波器 (BLF) 和加权最小二 乘方法(WLS)。第三节基于图像局部加权 TV 方法(adaptive $\mathrm{TV})$ 。第四节基于交替方向迭代的快速实现。第五节效果对 比与运算时间分析。

\section{2. 相关背景}

在数字图像处理中, 图像的分解一般分为基础层和细 节层。计算图像的基础层是将原图像降噪平滑的过程, 但 是图像的边界是一个锐化的区域, 这样就要求平滑操作必 须要保持原图像的边界, 并且要减少由平滑操作引入的光 晕现象。

近年来, 许多非线性保边界平滑方法被提出, 其中使用 最广泛是 BLF 方法。BLF 方法由 Tomasi 和 Manduchi[6] 最先提出。它是一种非线性滤波器, 在每个像素点的空间 邻域和值邻域都进行加权平均, 相当于在空间邻域和像素 值域分别进行 Gauss 滤波。

$$
\begin{gathered}
B L F(g)_{p}=\frac{1}{k_{p}} \sum_{q} G_{\sigma s}(\|p-q\|) G_{\sigma r}\left(\left\|g_{p}-g_{q}\right\| g_{q}\right) \\
k_{p}=\sum_{q} G_{\sigma s}(\|p-q\|) G_{\sigma r}\left(\left\|g_{p}-g_{q}\right\|\right)
\end{gathered}
$$

其中 $\mathrm{g}$ 是图像, 下标 $\mathrm{p}$ 和 $\mathrm{q}$ 表示像素点的空间位置, $k_{p}$ 是归一化因子。 $G_{\sigma s}$ 和 $G_{\sigma r}$ 表示 Gauss 滤波函数, $\sigma s$ 表示 空间邻域的核, $\sigma r$ 表示值域空间的核, 其 $\sigma r$ 的大小影响 滤波器对边界的敏感程度。Durand 和 Dorsey[7]给出 BLF 的快速算法, Paris[8]等人给出 BLF 的迭代表达式, 使 BLF 可以迭代计算。

尽管 BLF 结构简洁优雅, 并且对显著性边界保持以及 去掉小噪声都有很好的表现, 但是对任意尺度的细节提取 却有很大的限制。图 1 显示了这种限制。原图像在大区域 上是分片常像素值图像, 在每个大区域上随机加入不同尺 度的噪声, 即不同尺度的细节信息。然后分别使用 BLF, WLS 以及基于局部加权的 adaptive TV 方法进行平滑并且 提取不同尺度的细节信息。

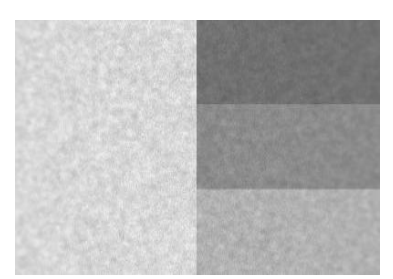

原图像(灰度图)

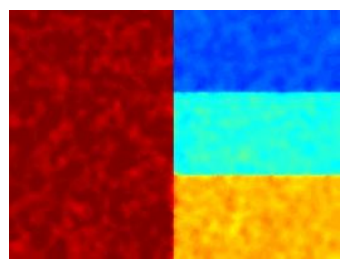

BLF(I)

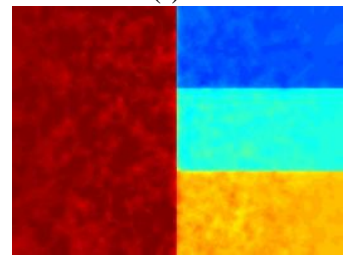

WLS(I)

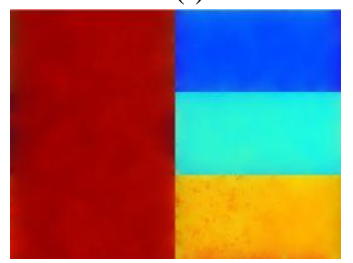

adaptive TV(I)

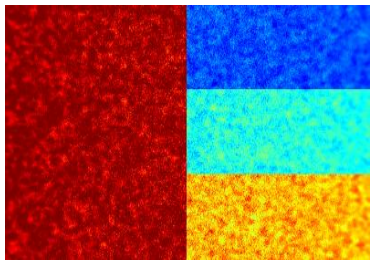

原图像

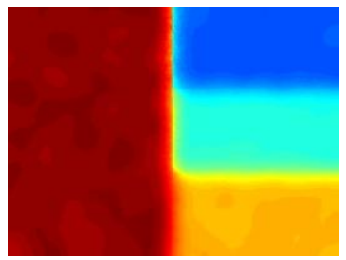

BLF(II)

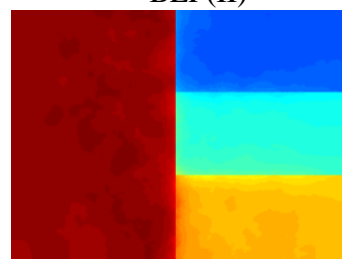

WLS(II)

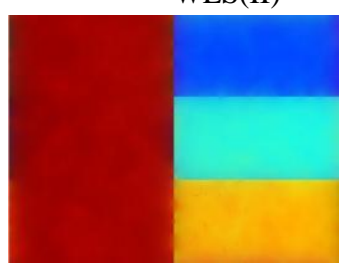

adaptive
图 1 BLF, WLS, adaptive TV 在大小两个尺度下的平滑效果

图 1 的 BLF(I) 和 BLF(II) 分别显示了在大小两个尺度下 提取细节的效果。BLF(I)可以看出, BLF 方法降低了小噪 声并且保持了显著性边界, 但是我们进一步加大尺度的时 候, 在去掉细节的同时, BLF(II)中的边界处开始出现光晕 效应。这是由于对于 $p$ 点处的像素值为 $g_{p}$, 当滤波操作时 需要对 $p$ 点的邻域做一个加权平均, 作为一个权值, 再在 值域空间做一个加权平均, 作为另外一个权值。当加大平 滑效果的时候, 就需要增大 $\sigma s$ 值, 考虑极限情况, 当 $\sigma s \rightarrow \infty$ 时, BLF 成为值域 Gauss 滤波器。但是为了保持 边界这就需要 $\sigma r$ 同时增大。当 $\sigma r \rightarrow \infty$ 时, BLF 成为 Gauss 线性滤波器。因此这种相互制约使得 BLF 并不适合 对任意尺度的细节进行提取。

为了对任意尺度的细节提取, Farbman[9]等人在 2007 年提出了加权最小二乘方法(WLS)。WLS 基本思想如下: 对于给定的原始图像 $\mathrm{g}$, 我们最终要得到一个图像 $u$, 其中 $u$ 满足两个方面: 其一就是每个对应点的像素值尽可能接 近原图像 $g$; 其二就是 $u$ 要比 $g$ 光滑, 这种光滑性的约束 体现在 $u$ 的梯度上。 
WLS 就是如下的一个极小值问题:

$$
\min \sum_{p}\left(\left(u_{p}-g_{p}\right)^{2}+\lambda\left(a_{x, p}(g)\left(\frac{\partial u}{\partial x}\right)_{p}^{2}+a_{y, p}(g)\left(\frac{\partial u}{\partial y}\right)_{p}^{2}\right)\right)
$$

这里的 $g$ 是原图像, $u$ 是平滑后图像, 下标 $p$ 表示像 素点的空间位置。对于数据项 $\left(u_{p}-g_{p}\right)^{2}$ 是保证 $\mathrm{u}$ 的每个对 应点的像素值尽可能接近 $g$; 对于正则项 $\left(a_{x, p}(g)\left(\frac{\partial u}{\partial x}\right)_{p}^{2}+a_{y, p}(g)\left(\frac{\partial u}{\partial y}\right)_{p}^{2}\right)$ 就是要求 $u$ 要尽可能光滑。 对于 $a_{x}$ 和 $a_{y}$ 则是对原图像 $g$ 的梯度检测函数, 是对原图像 $g$ 梯度分布的一种先验性检测 [10]。其具体定义如下:

$$
a_{x, p}(g)=\left(\left|\frac{\partial l}{\partial x}(p)\right|^{\alpha}+\varepsilon\right)^{-1}, a_{y, p}(g)=\left(\left|\frac{\partial l}{\partial y}(p)\right|^{\alpha}+\epsilon\right)^{-1}
$$

其中 $l$ 是像素值的对数值, $\alpha$ 是一个先验参数, 控制 着对图像 $g$ 的梯度分布的敏感程度, $\in$ 是一个小的正常数 (取 0.001)。

对于这个先验函数, 我们做以下简单的分析。首先对 原图像 $g$ 的像素值取对数, 使用 $\frac{\partial l}{\partial x}(p) 、 \frac{\partial l}{\partial y}(p)$, 压缩其像素 值的动态范围; 然后对于 $\left(a_{x, p}(g)\left(\frac{\partial u}{\partial x}\right)_{p}^{2}+a_{y, p}(g)\left(\frac{\partial u}{\partial y}\right)_{p}^{2}\right)$ 项来说 $a_{x}$ 和 $a_{y}$ 相当于 $\left(\frac{\partial u}{\partial x}\right)_{p}^{2} 、\left(\frac{\partial u}{\partial y}\right)_{p}^{2}$ 的线性组合的系数, 用来检 测原图像沿特定方向的梯度分布, 因此将各向同性的 $\left(\frac{\partial u}{\partial x}\right)_{p}^{2}+\left(\frac{\partial u}{\partial y}\right)_{p}^{2}$ 转化为各向异性的 $\left(a_{x, p}(g)\left(\frac{\partial u}{\partial x}\right)_{p}^{2}+a_{y, p}(g)\left(\frac{\partial u}{\partial y}\right)_{p}^{2}\right)$ 项, 以此来更好地刻画原图像的梯度分布。图 1 中的 WLS(I), WLS(II)方法在不同尺度下的保边界平滑能力。

\section{3. 基于局部加权的 adaptive TV}

WLS 方法使用正则项是 $\left(a_{x, p}(g)\left(\frac{\partial u}{\partial x}\right)_{p}^{2}+a_{y, p}(g)\left(\frac{\partial u}{\partial y}\right)_{p}^{2}\right)$, 使 用各向异性约束来刻画原图像的梯度分布, 本质上来讲是 $\mathrm{L}^{2}$ 范数的变种, 是一种特殊的各向异性范数。现在考虑 $\mathrm{L}^{1}$ 范数与 $\mathrm{L}^{2}$ 范数的区别, $\mathrm{L}^{2}$ 范数使用平方项是各向同性的, 其基本原理是基于 Gauss 分布; 而 $\mathrm{L}^{1}$ 范数则是各向异性的, 并且是基于 Laplacian 分布。在刻画梯度的稀疏性上, 各项 异性 $\mathrm{L}^{1}$ 范数更加的稀疏, 更能反映自然图像梯度的稀疏分 布。虽然这一约束能够保持图像的边缘信息, 但是对于某 些小尺度的细节, 以及孤立噪声点过于敏感。在使用该方 法时, 这些特征很有可能被当做成结构信息被保留下来, 这样导致结构提取的不准确。
为了克服这一缺点, 本文提出了基于局部加权的 adaptive TV 方法。使用 $\mathrm{L}^{1}$ 范数约束, 则 adaptive TV 就是 如下的一个极小值问题:

$$
\min \sum_{p}\left(\left(u_{p}-g_{p}\right)^{2}+\lambda w(g)\left(\left|\frac{\partial u}{\partial x}\right|_{p}+\left|\frac{\partial u}{\partial y}\right|_{p}\right)\right)
$$

这里的 $g$ 是原图像, $u$ 是平滑后图像, 下标 $p$ 表示像 素点的空间位置。对于数据项 $\left(u_{p}-g_{p}\right)^{2}$ 是保证 $u$ 的每个对 应点的像素值尽可能接近 $g$; 对于正则项 $\left(\left|\frac{\partial u}{\partial x}\right|_{p}+\left|\frac{\partial u}{\partial y}\right|_{p}\right)$ 则是 图像的全变差 $(\mathrm{TV})$ 梯度约束, 即各向异性 $\mathrm{L}^{1}$ 范数。当要求 $u$ 要尽可能光滑比 $g$ 光滑, 就是要求一个极小值问题。对 于 $w(g)$ 则是原图像梯度的检测函数, 是对原图像梯度分布 的一个先验。

对于 $w(g)$ 函数, 我们给出其构造:

$$
\begin{aligned}
& w(g)=\exp \left(-r(x)^{0.8}\right) \\
& r(x)=\frac{\left\|\sum y \in N_{h}(x) \nabla g(y)\right\|}{\sum y \in N_{h}(x)\|\nabla g(y)\|+0.5}
\end{aligned}
$$

其中 $\mathrm{g}$ 是原图像, $N_{h}(x)$ 是一个大小为 $h \times h$ 的窗口函 数, 0.5 是防止在 $h \times h$ 的窗口内常像素值而使得分母为 0 。 对于这样的一个先验函数 $w(g)$ 可以与 WLS 方法使用 的 $a_{x}$ 和 $a_{y}$ 先验函数进行对比分析。 $a_{x}$ 和 $a_{y}$ 考虑的是对原 图像梯度本身的检查, 是两个相邻点的像素差异; 而 $w(g)$ 考虑的是一个 $h \times h$ 的窗口内的梯度差异, 对于同一个窗口 内的像素点, 趋于 “平均” 化, 例如, 在平坦区域中, 有 小尺度细节处使得 $r(x)$ 的值比较大, $w(g)$ 的权值很小, 这 样可以有效的清除小尺度的细节, 以及孤立噪声的影响。 对于一个小的窗口区域内考虑, 可以有效的清除 “毛刺” 现象，是这个小区域更加各向同性。
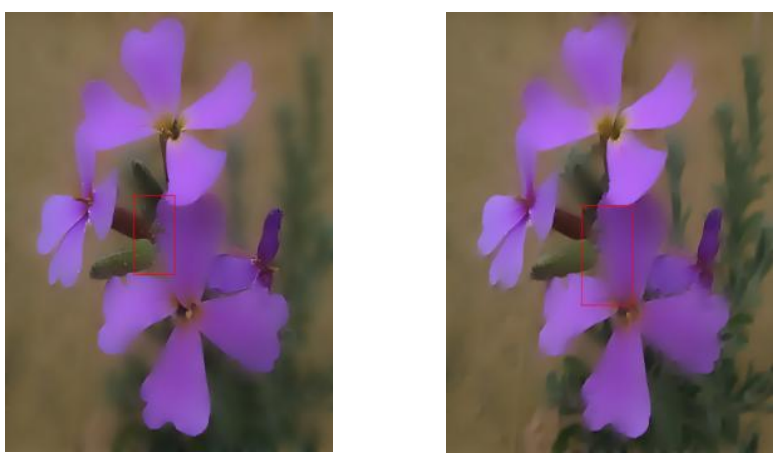

图 2: 左面图像使用 $a_{x}$ 和 $a_{y}$ 先验函数, 而右面图像使用 $w(g)$ 先 验函数, 图片放大红色区域内会看到噪点 
使用基于局部加权的 adaptive TV 方法和基础层和细 节层的定义, 我们可以得到图像的一个保边界平滑算法。 假设原始图像为 $g$, 构建其 $(\mathrm{k}+1)$ 阶分解, 使用符号 $u^{1} \cdots u^{k}$ 表示不同尺度下的各阶平滑图像, 这样我们就可以给出各 阶细节层的定义:

$d^{i}=u^{i-1}-u^{i}$, 其中 $i=1 \cdots k$ 并且定义 $u^{0}=g, u^{k}=b$

则原图像 $\mathrm{g}$ 也可以表示成如下关系:

$$
g=b+\sum_{i=1}^{k} d^{i}
$$

这样我们就得到了一个图像的多尺度分解方法, 并且 我们可以根据不同尺度的要求对任意的 $d^{i}$ 以及 $u^{i}$ 进行操 作。

\section{4. 算法实现}

对于基于局部加权的 adaptive TV 方法, 有原图像 $g \in R^{n^{2}}$, 梯度先验函数 $w(g)$, 我们得到平滑后的图像 $u$ :

$$
\min _{u}\|g-u\|_{2}^{2}+\lambda\|w \nabla u\|
$$

其中 $\nabla=\left(\frac{\partial}{\partial x}+\frac{\partial}{\partial y}\right)^{T}$ 。为了求解这个方法, 我们可以引入 辅助变量 $\omega=\omega_{1}, \omega_{2}$ 来逼近 $\frac{\partial u}{\partial x}, \frac{\partial u}{\partial y}$ 。

则问题(13)可转化为如下有约束优化问题

$$
\min \|g-u\|_{2}^{2}+\beta\left(\left\|\omega_{2}-\frac{\partial u}{\partial x}\right\|_{2}^{2}+\left\|\omega_{2}-\frac{\partial u}{\partial y}\right\|_{2}^{2}\right)+\lambda w(g)\left(\left\|\omega_{1}\right\|_{1}+\left\|\omega_{2}\right\|_{1}\right)
$$

其中当 $\beta \rightarrow \infty$, [Kernel estimation from salient structure for robust motion deblurring $]^{[11]}$ 给出了的解收玫到(9)的解的 收敛性保证。

式(14)计算过程可以通过交替迭代两个子问题来求解:

$$
\begin{aligned}
& \min _{\omega_{1}, \omega_{2}} \beta\left(\left\|\omega_{2}-\frac{\partial u}{\partial x}\right\|_{2}^{2}+\left\|\omega_{2}-\frac{\partial u}{\partial y}\right\|_{2}^{2}\right)+\lambda w(g)\left(\left\|\omega_{1}\right\|_{1}+\left\|\omega_{2}\right\|_{1}\right) \\
& \min \|g-u\|_{2}^{2}+\beta\left(\left\|\omega_{2}-\frac{\partial u}{\partial x}\right\|_{2}^{2}+\left\|\omega_{2}-\frac{\partial u}{\partial y}\right\|_{2}^{2}\right)
\end{aligned}
$$

对于问题(i), 根据收缩公式(Shrinkage formula)可以该 问题的闭解形式(11):

$$
\begin{aligned}
& \omega_{1}=\operatorname{sign}\left(\frac{\partial u}{\partial x}\right) \max \left(\left|\frac{\partial u}{\partial x}+\frac{\lambda w(g)}{2 \beta}\right|, 0\right) \\
& \omega_{2}=\operatorname{sign}\left(\frac{\partial u}{\partial y}\right) \max \left(\left|\frac{\partial u}{\partial y}+\frac{\lambda w(g)}{2 \beta}\right|, 0\right)
\end{aligned}
$$

对于问题(ii)是一个最小二乘问题, 我们可以通过频率 域的傅里叶变换 FFT 求得该问题的解:

$$
u=F^{-1}\left(\frac{F(I)+\beta\left(\overline{F\left(\partial_{x}\right)} \circ F\left(\omega_{1}\right)+\overline{F\left(\partial_{y}\right)} \circ F\left(\omega_{2}\right)\right)}{1+\beta\left(\overline{F\left(\partial_{x}\right)} \circ F\left(\partial_{x}\right)+\overline{F\left(\partial_{y}\right)} \circ F\left(\partial_{y}\right)\right)}\right)
$$

其中 $F(\bullet)$ 表示傅里叶变换, $F^{-1}(\cdot)$ 表示傅里叶逆变换, 符号 $\overline{F(\bullet)}$ 表示取复共轭, 符号“。”表示向量对应的分量可 运算。

通过使用快速 Fourier 变换(FFT)与其逆变换, 可以加 速基于局部加权的 adaptive TV 方法求解。最后重复迭代上 述的 $\omega$ 与 $u$, 我们最终可以得出保边界平滑后的图像 $u$ 。 给出计算机程序如下:

输入: 原图像为 $g$;

初始值: $u=g, \beta=1, \beta_{\text {min }}=1 / 2^{8}$

while $\beta>\beta_{\text {min }}$ do

Step 1: 通过式子(i)计算 $\omega$ 值.

Step 2: 通过式子(ii)计算 $u$ 值.

$\beta=\beta / 2$

end while

输出: $u$

\section{5. 算法对比}

对于 WLS 方法, 我们需要解线性方程组, 对于线性方 程的解法其时间复杂度约为 $\mathrm{o}\left(n^{2}\right)$; 对于 BLF 方法, 我们 使用 matlab 程序, 对应的源代码文件可以参考网站 http://www.cs.huji.ac.il/ danix/epd/附录所带的 matlab 程序。

与 WLS 方法相比, 局部加权的 adaptive TV 方法需要 求解两个子问题然后采用交替方向迭代算法(ADM), 对于 子问题(i), 我们可以由收缩方程直接得到其闭解形式(11); 对于子问题(ii), 则使用 FFT 变换及其 FFT 逆变换来加速求 解式子(12), 这里我们同样使用 matlab 自带的 FFT 变换函 数及其逆变换函数。

测试图像选取 4 幅并且编号如下:

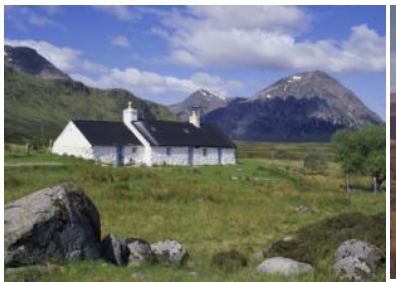

Test1

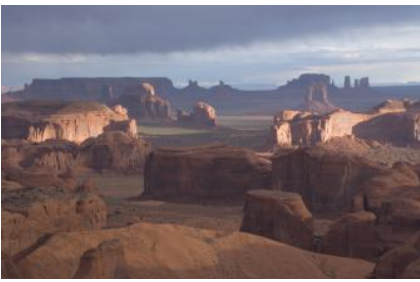

Test2 


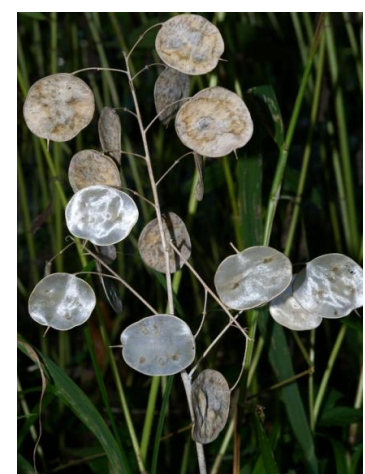

Test3

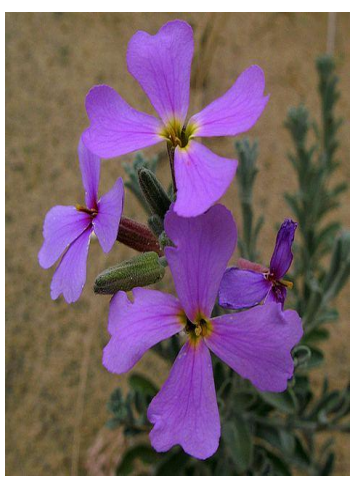

Test4
图 3

我们给出 BLF, WLS, adaptive TV 运算时间的对比表, 测试用的原图像 Test1-4。

注意: 表中程序为 matlab 程序, 运算时间均为运算 5 次取平均值, 单位为秒 (s), 取 4 位有效数字。

表： adaptive TV, WLS, BLF 运算时间的对比表

\begin{tabular}{|c|c|c|c|c|}
\hline & Test1 & Test2 & Test3 & Test4 \\
\hline adaptive TV & 5.074 & 4.872 & 3.325 & 4.231 \\
\hdashline WLS & 8.892 & 7.691 & 6.862 & 7.262 \\
\hdashline BLF & 6.482 & 6.182 & 5.048 & 5.236 \\
\hline
\end{tabular}

最后, 我们给使用 WLS 方法和基于局部加权的 adaptive TV 方法的保边界平滑效果示例。

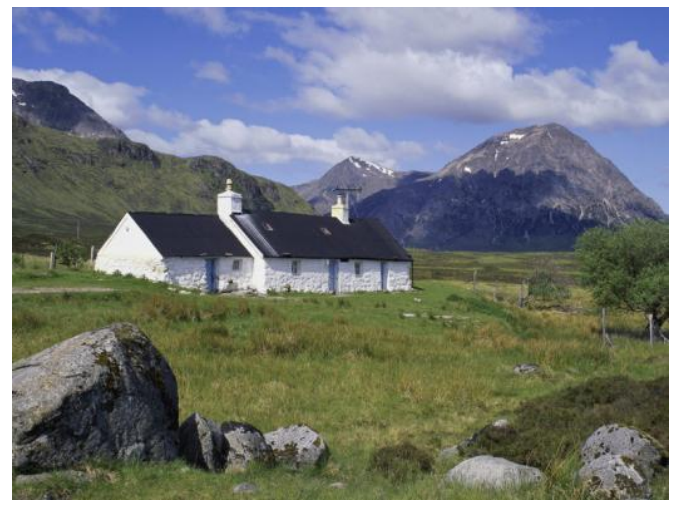

Test1

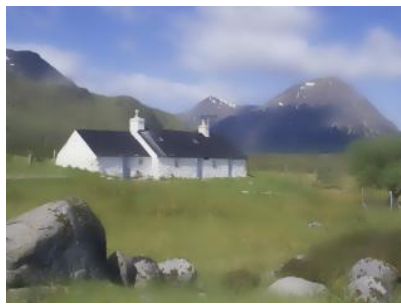

WLS

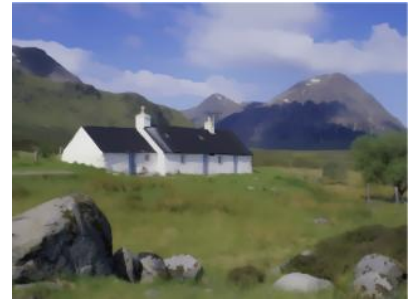

adaptive TV

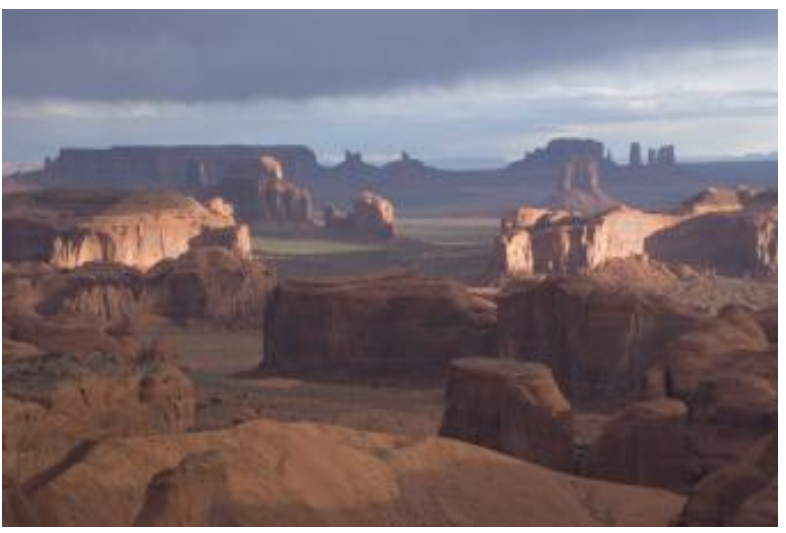

Test2

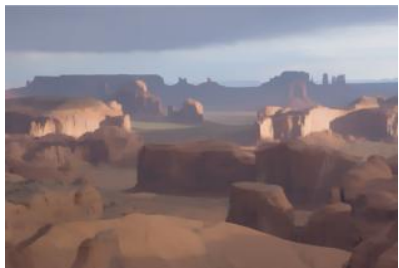

WLS

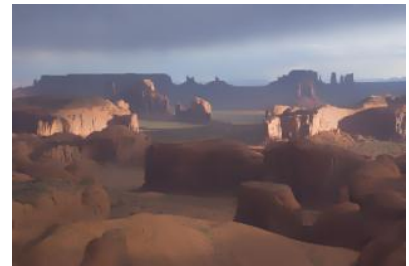

adaptive TV

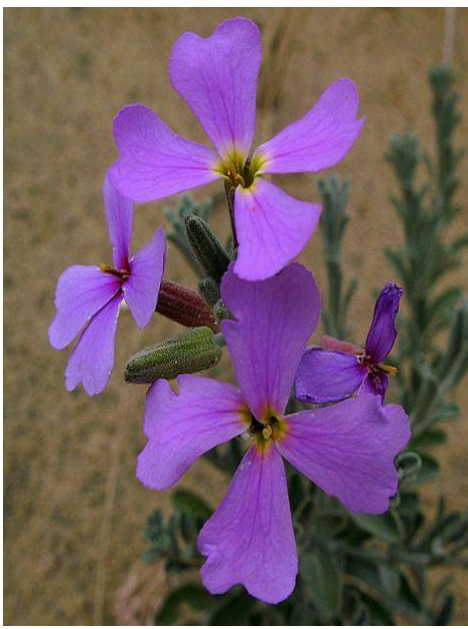

Test3

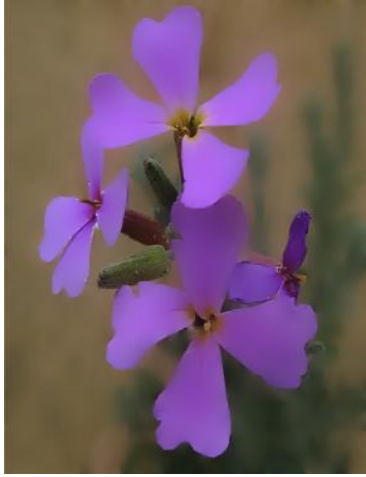

WLS adaptive TV

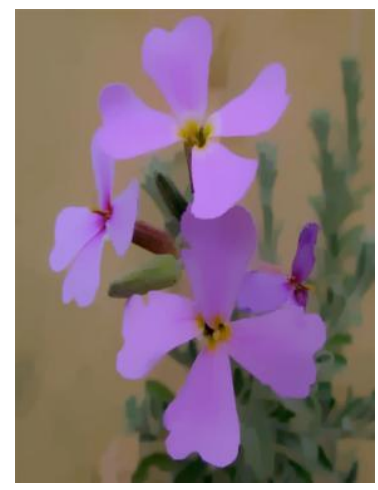




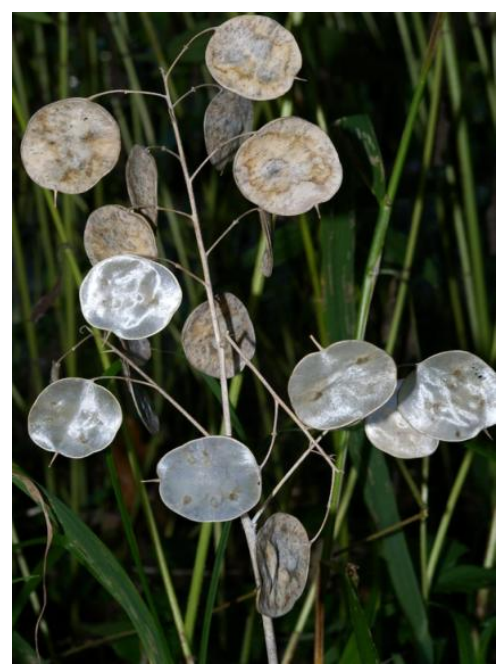

Test4

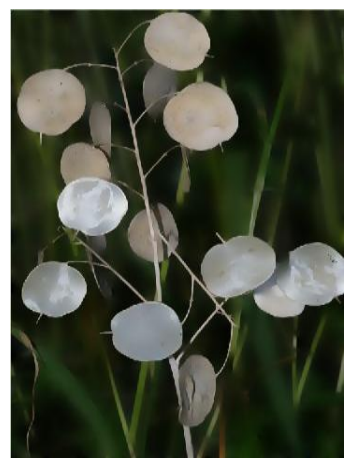

WLS

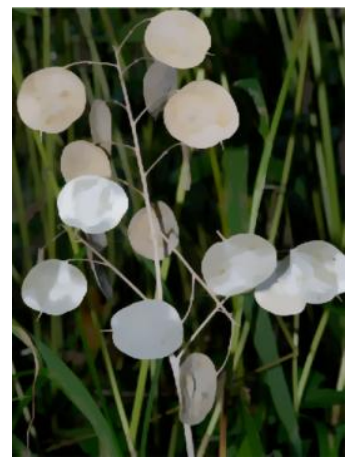

adaptive TV
图 4 测试图像平滑效果示例

基于局部加权的 adaptive TV 与 WLS 方法相比, 在刻 画原图像的梯度上有着明显的优势, 对原图像梯度的稀疏 性刻画更好, 如 test1,test4 中明显的可以看到对”显著性”的 边界保持, 同时对细节信息进行更有效的平滑, 如 test 1 中 的草坪, 石块内的纹理, 以及 test 4 中的背景的绿叶。由于 使用基于了局部加权, adaptive TV 可以减少直接使用 TV 项产生的孤立噪声的影响, 即 adaptive TV 方法可以有效的 清除 “毛刺” 现象, 如 test3 花瓣中没有产生孤立的噪声点, 使得 test3 有更好的平滑效果。

\section{6. 结论}

我们提出了一个更有效的算法用于任意尺度图像的保 边界平滑。利用区域化的梯度检测函数和使用各向异性范 数( $\mathrm{TV}$, 即各向异性 $\mathrm{L}^{1}$ 范数) 来刻画原图像的梯度分布并以 此来保持图像的边界。实验表明, 与传统保边界平滑算法 如双边滤波器(BLF)相比, 基于局部加权的 adaptive TV 可 以用于任意尺度下的图像分解, 边界处也可以减小由于振 铃效应所带来的光晕现象以及梯度反转现象; 与加权最小
二乘方法(WLS)相比，本文提出的这种基于局部加权的图 像的全变差约束可以更好的刻画梯度分布保持边界, 同时, 基于局部加权的 adaptive TV 方法的时间约是 WLS 方法的 $50 \%$ ，在计算时间上与 WLS 方法相比有明显优势。在运算 时间和平滑效果上基于局部加权的 adaptive TV 方法都是 一种非常优秀的保边界平滑算法。

\section{参考文献(References)}

[1] TUMBLIN, and J., TURK, "A boundary hierarchy for detail-preserving contrast reduction." In Proc. ACM SIGGRAPH 99, A. Rockwood, Ed.,ACM, 83-90.

[2] PETSCHNIGG, G., SZELISKI, R., AGRAWALA, M., COHEN, M., HOPPE, H., and TOYAMA, K. 2004. "Digital photography with flash and no-flash image pairs." ACM Trans. Graph. 23, 3 (Au- gust), 664-672.

[3] OH, B. M., CHEN, M., DORSEY, J., and DURAND, F. "Image-based modeling and photo editing."'In Proc. ACM SIGGRAPH 2001, ACM, E. Fiume, Ed., 433-442.

[4] BURT, P., and ADELSON, E. H. 1983. "The Laplacian pyramid as a compact image code." IEEE Trans. Comm. 31, 532-540.

[5] ZEEV FARBMAN, RAANAN FATTAL, and DANI LISCHINSKI, RICHARD. "Edge-Preserving Decompositions for Multi-Scale Tone and Detail Manipulation." ACM Trans. Graph. 25(3), 787-794 (2008).

[6] TOMASI, C., and MANDUCHI, R. "Bilateral filtering for gray and color images.” In Proc. ICCV '98, IEEE Computer So- ciety, 839-846.

[7] .DURAND, F., and DORSEY, J. 2002. "Fast bilateral filtering for the display of high-dynamic-range images." ACM Trans. Graph. 21, 3 (July), 257-266.

[8] PARIS, S. 2007. "A gentle introduction to bilateral filtering and its applications." In ACM SIGGRAPH 2007 courses, Course 13.

[9] FATTAL, R., LISCHINSKI, D., and WERMAN, M. "Gradient domain high dynamic range compression." ACM Trans. Graph. 21, 3 (July), 249-256.

[10] ZEEV FARBMAN, RAANAN FATTAL, and DANI LISCHINSKI, RICHARD. "Edge-Preserving Decompositions for Multi-Scale Tone and Detail Manipulation." ACM Trans. Graph. 25(3), 787-794 (2008).

[11] J. Pan, R. Liu, Z. Su, and X. Gu, "Kernel estimation from salient structure for robust motion deblurring,"2012. [Online]. Available: http://arxiv.org/abs/1212.1073 